\title{
Frequency of Going Out and Locomotive Syndrome Among Japanese Female Elderlies
}

\author{
Fumie Okada ${ }^{1,2}$, Satoshi Toyokawa ${ }^{3}$, Takehiko Kaneko ${ }^{1} \&$ Tadashi Furuhata $^{1}$ \\ ${ }^{1}$ Department of Health and Nutrition, Wayo Women's University, Chiba, Japan \\ ${ }^{2}$ Koyo Nursing Nutrition College, Ibaraki, Japan \\ ${ }^{3}$ Graduate School of Medicine, The University of Tokyo, Tokyo, Japan \\ Correspondence: Satoshi Toyokawa, Graduate School of Medicine, The University of Tokyo, 7-3-1 Hongo, \\ Bunkyo, Tokyo, 113-0033, Japan. Tel: 03-5841-3494. E-mail: t-satoshi@umin.ac.jp
}

Received: October 18, 2019 Accepted: November 22, 2019 Online Published: December 15, 2019

doi:10.5539/gjhs.v12n1p80 URL: https://doi.org/10.5539/gjhs.v12n1p80

\begin{abstract}
Background: Japan is the world's leading super-aged society, which makes locomotive syndrome an urgent issue. Because increasing the frequency of going out is considered a practical primary preventive measure against locomotive syndrome, we examined the relationship between the frequency of going out and locomotive syndrome in elderly females in Japan.

Methods: The subjects were 8,027 females from 46 prefectures in Japan who were living at home and aged 65 and older as of November 1, 2012. The study period was from November 1 to December 31, 2012. The survey was implemented by distributing questionnaires, as well as conducting face-to-face interviews. Odds ratios were obtained using logistic regression models with locomotive syndrome as the dependent variable.
\end{abstract}

Results: Eight thousands twenty seven females were analyzed in this study. There was a significant difference in the prevalence of locomotive syndrome depending on the frequency of going out $(p<0.001)$ as the prevalence of locomotive syndrome decreased as the frequency of going out increased. When the results were adjusted for gender, the frequency of going out, age, use of national nursing-care insurance services, household composition, severity of obesity, and self-rated health, the prevalence of locomotive syndrome was high in those whose frequency of going out was "twice or less a week" (Odds ratio: 1.41, 95\% Confidence interval 1.20-1.64).

Conclusions: The results suggest that it is possible to prevent locomotive syndrome by encouraging elderly people to maintain and increase their frequency of going out.

Keywords: elderly females living at home, locomotive syndrome, sarcopenia, going out

\section{List of Abbreviations}

OR: Odds Ratio, CI: Confidence Interval, BMI: Body Mass Index, MET: Metabolic Equivalent

\section{Background}

Japan is the world's leading super-aged society, but there is a marked regional difference in healthy life expectancy (Ministry of Health, Labour and Welfare, 2016; Ministry of Health, Labour and Welfare, 2015a). Prevention of functional decline in elderly people is an issue attracting increasing attention (Ministry of Health, Labour and Welfare, 2016). Locomotive syndrome is a predisposing factor for the decline of motor abilities (Nakamura, 2008). Locomotive syndrome refers to conditions under which elderly people require nursing care or are bedridden, or high-risk conditions under which they may soon require nursing care or become bedridden, owing to the functional decline of locomotive organs, such as bones, joints, and muscles, that support and allow us to move our body (Ministry of Health, Labour and Welfare, 2016; Nakamura, 2008; Nakamura, 2009). The concept of locomotive syndrome includes sarcopenia, which is a clinical manifestation of frailty (Kazuya, 2015). Although the prevalence of locomotive syndrome nationwide has not yet been determined by a survey, a survey conducted on middle-aged and elderly people in a local community in Japan found it was prevalent in $21.2 \%$ of males and $35.6 \%$ of females of this community. Thus, it is estimated that 47 million people throughout the country have at least one of the following: knee osteoarthritis, lumbar spondylosis, and osteoporosis (Yoshimura 2012). These diseases are a physical burden on elderly people. The utility of surgical treatment is limited because surgical 
treatment is too invasive to treat diseases that are not serious in most patients. Further, clinical intervention with supplements produces limited effects. Therefore, the primary preventive measure against locomotive syndrome involves weight control and physical activity, as Rose's population strategy of prevention suggests (Rose, 2008).

Exercise with a light physical load is suggested to prevent locomotive syndrome (Japanese Orthopaedic Association, 2015). However, considering the high prevalence of this syndrome, it is difficult to increase the rate of participation in exercise classes. Thus, this approach is not likely to allow collective action. Furthermore, from the perspective of a population strategy, a preventive measure against locomotive syndrome should involve behavioral changes that can be implemented in everyday life. Elderly people may easily agree with the idea of increasing the frequency of going out and they may make implement this measure. However, to the best of our knowledge, little is known about the relationship between the frequency of going out and locomotive syndrome. Therefore, we studied this relationship within elderly females in Japan.

\section{Methods}

\subsection{Subjects and Survey Procedures}

The study target population was 15,200 people living in all prefectures, except for one prefecture, Tokyo, and who were living at home and aged 65 or older as of November 1, 2012. The study period was from November 1 to December 31, 2012. The survey was implemented by distributing a questionnaire titled "Survey on Dietary Environment in Relation to Difficulty in Shopping and Current Health Conditions of Household Elderly" (Okada, 2012), whereby trained dietary health-promoting volunteers randomly sampled their elder neighbors as subjects and conducted face-to-face interviews in the subjects' homes to help subjects in filling out the questionnaire.

\subsection{Questionnaire Items}

The questionnaire consisted of 31 questions about daily life, including health conditions, grocery shopping, eating habits, and the use of food delivery services (Okada, 2012). Those determined to have locomotive syndrome answered "yes" to the question about currently having diseases of the bones and joints being treated. Age, gender, household composition, major source of income, need for nursing care, and severity of obesity were used as adjustment factors. Based on the report on the elderly published by the Planning Committee for Health Japan 21 (for the second term) and prepared by the Ministry of Health, Labor and Welfare (2012), the severity of obesity was classified into one of the following three categories according to body mass index (BMI) $\left(\mathrm{kg} / \mathrm{m}^{2}\right)$ (as calculated from height and weight): $<20$, underweight; $20-25$, normal; and $\geq 25$, obese.

Based on the answers to the question about household composition, the subjects were classified into three groups: "single household," "married-couple household," and "living with children." Those who selected "others" were included in the group "living with children." Because most of them lived with their consanguine family. The subjects were also asked about the use of national nursing-care insurance services and their required care and support levels. Results for the use of national nursing-care insurance services were analyzed. Moreover, the subjects were classified into the following two groups based on their answer to the question about their current major source of income: "pension" or "work (including self-owned business)." Those who selected "others" were included in the group "work" because this meant they had multiple income sources.

Based on their answer to the question about the frequency of going out (including shopping), the subjects were classified into the following four groups: "almost every day," "three or four times a week," "once or twice a week," and "once to three times a month or rarely."

Based on the answers to the question about self-rated health, the subjects were classified into the following five groups: "good," "rather good," "neither good nor poor," "rather poor," and "poor."

\subsection{Statistical Analyses}

Analyses were conducted only on females. The distribution of the frequency of going out and that of adjustment factors were described by the presence or absence of locomotive syndrome and significance was determined by the chi-squared test. Age is presented as a mean and standard deviation (SD) and the significance of differences was determined using student's t-test. The odds ratio (OR) and 95\% confidence interval (CI) were obtained using logistic regression models with locomotive syndrome as the dependent variable. The statistical models used were as follows: a crude model that included factors alone, a multivariate model that included frequency of going out, and confounding factors, which included age, use of national nursing-care insurance services, household composition, and severity of obesity (Model 1) (Okada, 2015; Okada, 2017) . To adjust the reverse effects induced by serious forms of locomotive syndrome inhibiting the elderly from going out, a multivariate model that included self-rated health was performed in addition to the covariates included in Model 1 (Model 2). Statistical 
significance was defined as $p<0.05$. Statistical tests were performed using Stata 13 (Stata Copr, 2013).

\section{Results}

Although 15,200 was the target number of subjects, 12, 782 responded to the survey (crude response rate: $84.1 \%$ ). Valid responses without missing data were obtained from 10,226 respondents (valid response rate: $67.2 \%$ ). Among them, 8,027 were female and were, thus, analyzed in this study.

The mean age of the subjects with locomotive syndrome (78.1 \pm 6.5 years) was significantly higher than that of those without locomotive syndrome $(75.3 \pm 6.9$ years; $p>0.001)$ (Table 1$)$. The BMI of the subjects with locomotive syndrome (22.8 \pm 3.2$)$ was significantly higher than that of those without locomotive syndrome $(22.4 \pm 3.0 ; p>0.001)$. There was a significant difference in the prevalence of locomotive syndrome depending on BMI $(p<0.001)$; the prevalence of locomotive syndrome was higher in the group with BMIs of $\geq 25(1.31,1.15-1.50)$ and was lower in the group with BMIs of $<20(0.81,0.70-0.93)$. There was a significant difference in the prevalence of locomotive syndrome depending on the frequency of going out $(p<0.001)$; the prevalence of locomotive syndrome decreased as the frequency of going out increased. The prevalence of locomotive syndrome was higher in those who used national nursing-care insurance services $(p<0.001)$, and in those who lived in single households. There was also a significant difference in the prevalence of locomotive syndrome depending on self-rated health levels, as the prevalence of locomotive syndrome decreased as the self-rated health levels increased.

Table 1. Distribution of frequency of going out and that of adjustment factors for presence or absence of locomotive syndrome

\begin{tabular}{|c|c|c|c|c|c|}
\hline \multirow{3}{*}{ Age } & \multirow{2}{*}{\multicolumn{2}{|c|}{$\begin{array}{l}\text { With locomotive syndrome } \\
N=5,929\end{array}$}} & \multirow{2}{*}{\multicolumn{2}{|c|}{$\begin{array}{l}\text { Without locomotive syndrome } \\
N=2,098\end{array}$}} & \multirow{3}{*}{$\begin{array}{l}P \text { value } \\
<0.001\end{array}$} \\
\hline & & & & & \\
\hline & 75.29 & \pm 6.95 & 78.12 & \pm 6.54 & \\
\hline \multicolumn{6}{|l|}{ BMI levels } \\
\hline$<20$ & 1,244 & $74.9 \%$ & 417 & $25.1 \%$ & \multirow{3}{*}{$<0.001$} \\
\hline $20-25$ & 3,663 & $75.2 \%$ & 1,208 & $24.8 \%$ & \\
\hline$\geqslant 25$ & 1,022 & $68.4 \%$ & 473 & $31.6 \%$ & \\
\hline \multicolumn{6}{|l|}{ Household } \\
\hline Single household & 2,083 & $68.6 \%$ & 954 & $31.4 \%$ & \multirow{3}{*}{$<0.001$} \\
\hline Married-couple household & 2,084 & $79.3 \%$ & 545 & $20.7 \%$ & \\
\hline Living with children/Others & 1,762 & $74.6 \%$ & 599 & $25.4 \%$ & \\
\hline \multicolumn{6}{|l|}{ Pension } \\
\hline Pension & 5,334 & $73.6 \%$ & 1,915 & $26.4 \%$ & \multirow{2}{*}{$<0.081$} \\
\hline Other than pension & 595 & $76.5 \%$ & 183 & $23.5 \%$ & \\
\hline \multicolumn{6}{|c|}{ Use of national nursing-care insurance services } \\
\hline Not using & 5,464 & $76.5 \%$ & 1,683 & $23.5 \%$ & \multirow{2}{*}{$<0.001$} \\
\hline Using & 465 & $52.8 \%$ & 415 & $47.2 \%$ & \\
\hline \multicolumn{6}{|l|}{ Self-rated health } \\
\hline Good & 1,380 & $90.3 \%$ & 149 & $9.7 \%$ & \multirow{5}{*}{$<0.001$} \\
\hline Rather good & 1,742 & $78.0 \%$ & 491 & $22.0 \%$ & \\
\hline Neither good nor poor & 2,209 & $72.7 \%$ & 831 & $27.3 \%$ & \\
\hline Rather poor & 544 & $49.8 \%$ & 549 & $50.2 \%$ & \\
\hline Poor & 54 & $40.9 \%$ & 78 & $59.1 \%$ & \\
\hline \multicolumn{6}{|l|}{ Frequency of going out } \\
\hline Almost every day & 1,672 & $81.5 \%$ & 379 & $18.5 \%$ & \multirow{4}{*}{$<0.001$} \\
\hline Three or four times a week & 1,938 & $76.2 \%$ & 604 & $23.8 \%$ & \\
\hline Once or twice a week & 1,442 & $67.8 \%$ & 685 & $32.2 \%$ & \\
\hline Less than three times a month & 877 & $67.1 \%$ & 430 & $32.9 \%$ & \\
\hline
\end{tabular}


Table 2. Odds ratios of locomotive syndrome depending on frequency of going out in elderly females

\begin{tabular}{|c|c|c|c|c|c|c|c|c|c|c|c|c|}
\hline \multirow[b]{3}{*}{ Age } & \multicolumn{4}{|c|}{ Crude OR } & \multicolumn{4}{|c|}{ Adjusted OR (model1) } & \multicolumn{4}{|c|}{ Adjusted OR2 (model2) } \\
\hline & \multirow{2}{*}{$\begin{array}{c}\text { OR } \\
1.06\end{array}$} & \multirow{2}{*}{$\begin{array}{l}95 \% \\
\text { level }\end{array}$} & \multicolumn{2}{|r|}{ confidence } & \multirow{2}{*}{$\begin{array}{c}\text { OR } \\
1.04\end{array}$} & \multirow{2}{*}{$\begin{array}{l}95 \% \\
\text { level }\end{array}$} & \multicolumn{2}{|c|}{ confidence } & \multirow{2}{*}{$\begin{array}{l}\text { OR } \\
1.03\end{array}$} & \multirow{2}{*}{$\begin{array}{l}95 \% \\
\text { level }\end{array}$} & \multicolumn{2}{|c|}{ confidence } \\
\hline & & & - & 1.07 & & & - & 1.05 & & & - & 1.04 \\
\hline \multicolumn{13}{|l|}{ BMIlevels } \\
\hline$<20$ & 1.00 & & & & 1.00 & & & & 1.00 & & & \\
\hline $20-25$ & 1.02 & 0.89 & - & 1.16 & 0.84 & 0.74 & - & 0.97 & 0.81 & 0.70 & - & 0.93 \\
\hline$\geq 25$ & 1.40 & 1.24 & - & 1.59 & 1.41 & 1.24 & - & 1.61 & 1.31 & 1.15 & - & 1.50 \\
\hline \multicolumn{13}{|l|}{ Household } \\
\hline Living with children/Others & 1.00 & & & & 1.00 & & & & 1.00 & & & \\
\hline Single household & 1.35 & 1.19 & - & 1.52 & 1.19 & 1.04 & - & 1.35 & 1.19 & 1.04 & - & 1.36 \\
\hline Married-couple household & 0.77 & 0.67 & - & 0.88 & 0.96 & 0.83 & - & 1.10 & 0.95 & 0.82 & - & 1.10 \\
\hline \multicolumn{13}{|l|}{ Major source of income } \\
\hline Pension & 1.00 & & & & 1.00 & & & & 1.00 & & & \\
\hline Other than pension & 1.17 & 0.98 & - & 1.39 & 0.99 & 0.83 & - & 1.20 & 0.94 & 0.78 & - & 1.13 \\
\hline \multicolumn{13}{|c|}{ Use of national nursing-care insurance services } \\
\hline Not using & 1.00 & & & & 1.00 & & & & 1.00 & & & \\
\hline Using & 2.90 & 2.51 & - & 3.34 & 1.97 & 1.69 & - & 2.30 & 1.48 & 1.26 & - & 1.74 \\
\hline \multicolumn{13}{|l|}{ Self-rated health } \\
\hline Good & 1.00 & & & & & & & & 1.00 & & & \\
\hline Rather good & 2.61 & 2.14 & - & 3.18 & & & - & & 2.38 & 1.95 & - & 2.90 \\
\hline Neither good nor poor & 3.48 & 2.89 & - & 4.20 & & & - & & 3.08 & 2.55 & - & 3.72 \\
\hline Rather poor & 9.35 & 7.60 & - & 11.49 & & & - & & 6.78 & 5.48 & - & 8.40 \\
\hline Poor & 13.38 & 9.09 & - & 19.68 & & & - & & 10.26 & 6.90 & - & 15.25 \\
\hline \multicolumn{13}{|l|}{ Frequency of going out } \\
\hline Almost every day & 1.00 & & & & 1.00 & & & & 1.00 & & & \\
\hline Three or four times a week & 1.37 & 1.19 & - & 1.59 & 1.26 & 1.09 & - & 1.46 & 1.14 & 0.98 & - & 1.32 \\
\hline Once or twice a week & 2.10 & 1.81 & - & 2.42 & 1.67 & 1.44 & - & 1.94 & 1.41 & 1.20 & - & 1.64 \\
\hline $\begin{array}{l}\text { Less than three times a } \\
\text { month }\end{array}$ & 2.16 & 1.84 & - & 2.54 & 1.46 & 1.23 & - & 1.73 & 1.13 & 0.95 & - & 1.35 \\
\hline Hosmer-Lemeshow test & & & & & $\mathrm{p}=0.0$ & & & & $\mathrm{p}=0.5$ & & & \\
\hline
\end{tabular}

Logisitic regression, OR: Odds ratio;

Covariates; (model1) Age, BMI level, Use of national nursing-care insurance services, Household, Pension, Frequency of going out;

(model2) model1 + Self-rated heath.

The crude OR was highest in subjects whose frequency of going out was less than three times a month (OR: 2.16, 95\% CI: 1.84-2.54), followed by subjects whose frequency of going out was once or twice a week (OR: 2.10, 95\% CI: 1.81-2.54), and lastly subjects whose frequency of going out was three or four times a week (OR: 1.37, 95\% CI: 1.19-1.59) (Table 2). The ORs between the frequency of going out and locomotive syndrome decreased as the frequency of going out increased. When adjusted for age, required care and support levels, household composition, and major source of income, the OR in subjects whose frequency of going out was less than three times a month decreased to 1.46 (95\% CI: 1.23-1.73). When adjusted for self-rated health, the OR decreased to 
nearly 1 (OR: $1.03,95 \% \mathrm{CI}: 1.03-1.04)$. In the multivariate analysis, a significantly high OR was observed in subjects whose frequency of going out was once or twice a week (OR: 1.41, 95\% CI: 1.20-1.64). A significant relationship was observed in subjects who lived in single households (OR: 1.19,95\%CI: 1.04-1.36) and who used national nursing-care insurance services (OR: 1.48, 95\%CI: 1.26-1.74). As for self-rated health, a significant OR for high risk was observed in subjects who selected "rather good," (OR: 2.38, 95\%CI: 1.95-2.90), compared with subjects who selected "good." The OR increased as self-rated health worsened. As a result of the HosmerLemeshow test, Model 1 showed a significant difference $(p=0.023)$, indicating the instability of the model, while Model 2 did not show a significant difference $(p=0.526)$, indicating the stability of the model.

\section{Discussion}

We examined the relationship between the frequency of going out and locomotive syndrome in 8,027 elderly females living at home in Japan. In the bivariate analysis, the prevalence of locomotive syndrome increased as the frequency of going out decreased. In the multivariate analysis using Model 1, the OR was 1.46 in subjects whose frequency of going out was the lowest (less than three times a month). However, in the multivariate analysis using Model 2, which included self-rated health, the OR became close to 1, namely, 1.13. This may be because self-rated health levels decreased owing to locomotive syndrome and other preexisting diseases that affect the frequency of going out. The relationship between cause and effect predicted in Model 1 seemed to be adjusted by the reverse relationship in Model 2, namely a decrease in the frequency of going out due to locomotive syndrome. Further, the OR was 1.41 in subjects whose frequency of going out was once or twice a week in Model 2, which included self-rated health; however, the prevalence of locomotive syndrome was still significantly high in this group. This difference in OR between subjects whose frequency of going out was once or twice a week, and those whose frequency of going out was less than three times a month, may reflect the fact that the prevalence of locomotive syndrome was high in females living at home and whose frequency of going out was once or twice a week but whose self-rated health had not declined.

The preventive effect of the high frequency of going out on locomotive syndrome can be explained in relation to the network of locomotive organs, such as bones, joints, muscles, and the peripheral and central nervous systems. The fundamental means of going out is erect walking with both legs. This way of walking is energy-efficient but unstable because the duration of a one-leg stance accounts for about 3/4 of one walking cycle (Nakamura 2014). Therefore, going out by walking becomes possible only through precise control of the entire network of locomotive organs.

First, bone metabolism may contribute to the prevention of locomotive syndrome. Metabolic modeling of bones occurs during the stage of growth at which bone formation exceeds bone resorption, resulting in changes in the shape and size of bones. In elderly people, however, bone mass gradually decreases with age, and is dependent on the peak bone mass achieved during the stage of growth, followed by the rate of bone loss from then on. Further, it decreases sharply during menopause caused by the deficiency of estrogen. In addition to diets containing sufficient amounts of protein, calcium, vitamin $\mathrm{D}$, and potassium, exercises in which force of gravity is vertically applied to bones, such as fast walking and running, are recommended to prevent bone loss (Japan Osteoporosis Society, the Japanese Society for Bone and Mineral Research, \& Japan Osteoporosis Foundation, 2015). Thus, bone mass and bone quality decrease as the frequency of going out decreases, resulting in a predisposition to osteoporosis and an increased risk of fracture.

The periosteum is found at the bone periphery. The periosteum contains blood vessels and nerves, and envelopes the bone completely. When a muscle is moved, the bone that serves as a strut is slightly damaged. Through bone metabolism, the damaged bone is replaced by new bone produced by osteoblasts. Similar to the process of skin repair, calcium, a bone component, accumulates to repair damage to the bone. Bone strength increases as this "repair action" is repeated. In this way, exercises to train muscles also stimulate the bone covered by muscles, contributing to the maintenance of bone strength. Therefore, going out leads to improvement of bone and muscle strength, and is also an effective measure against sarcopenia. In contrast, it seems that a decrease in the frequency of going out leads to a decrease in bone and muscle strength. When bones are weakened to the point of osteoporosis and other clinical conditions, locomotive and walking functions are impaired owing to limited range of joint motion, which results in gait disorders. Gait disorder and a decrease in the frequency of going out then further contribute to diseases of the bones, articular cartilages, and intervertebral discs. A decrease in the frequency of going out, thus, may begin limiting daily living activities and enable the conditions that require nursing care given locomotive syndrome (Ministry of Health, Labour and Welfare, 2015b).

For humans who walk in an unstable two-legged posture, joints are components of a network of locomotive organs. One of the typical joint problems is osteoarthritis. Patients with osteoarthritis experience pain and stiffness of the 
joints, limited range of joint motion, and morphological degeneration of joints, as a result of the degeneration of articular cartilage. Further, osteoarthritis contributes to locomotive syndrome. Age, race, occupation, obesity, and previous injuries are reported as risk factors for osteoarthritis (Kawagachi 2014). Most of the cellular and molecular mechanisms of this clinical condition have remained unclarified and there has been no definitive or curative therapy for osteoarthritis. Besides weight control and the ability to maintain the pelvis in a normal position, gait improvement is also important (Takahashi, 2003) and may be related to the frequency of going out.

Skeletal muscles, generally called muscles, are also components of the network of locomotive organs. Muscle mass decreases with age, and this degenerative loss of muscle mass is called sarcopenia (Kazuya, 2015). Sarcopenia begins around the ages ranging from 25 to 30. Inactivity is considered to be the main cause of sarcopenia, but its mechanism has not yet been fully clarified. The progress of sarcopenia can be suppressed by training aimed at increasing muscle strength and muscle mass, which may underlie the relationship between the frequency of going out and locomotive syndrome.

Physical activity is important for the network components of locomotive organs. A Korean cross-sectional study suggested that middle-aged adults who met with their neighbors 1 to 3 times a week were less likely to be overweight than those with once-a-year meeting frequency (Noh, 2016). A Japanese longitudinal study showed that an active and socially integrated lifestyle in later life might play a major role in maintaining a healthy status (Sakurai, 2018). "Physical Activity Reference for Health Promotion 2013" recommends 10 metabolic equivalent (MET) hours/week of physical activity regardless of intensity as the reference physical activity (activities of daily living and exercises) for people aged 65 and older. Namely, people aged 65 and older are encouraged to do any form of physical activity, except those in which they are continuously lying down or sitting, for 40 minutes every day. Going out seems to be a form of sustainable physical activity (Ministry of Health, Labour and Welfare, 2013).

\subsection{Limitations of this Study}

The present study had several limitations. First, the study was a cross-sectional study; thus, no causal interpretations can be made. Second, there may be a lack of accuracy of the information obtained because the answers to the questionnaire were based on memories of the study subjects and susceptible to recall bias. Third, the subjects were not randomly selected from the general population. Finally, all measurements, including the frequency of going out, were done without assessing reliability and validity.

\section{Conclusion}

The prevalence of locomotive syndrome is high among females aged 65 and older living at home in Japan, and whose frequency of going out was twice or less a week, and low among those who went out almost every day.

\section{Ethics Approval, Consent to Participate}

Since the subjects were asked about their households and personal affairs, we took all possible measures to protect and manage the subjects' personal data and to dispel fears regarding privacy. The interviewers obtained consent from subjects after fully explaining the purpose and methods of this study and that the participation in this survey was voluntary. The survey and analysis were conducted with the approval of the Ethics Committee of Tokiwa University (No. 100025).

\section{Consent for Publication}

Not applicable.

\section{Availability of Data and Materials}

The research datasets for this paper are unsuitable for public deposition due to ethical restrictions. However, the datasets used and/or analyzed in the present study are available from the corresponding author on reasonable request.

\section{Funding}

No funding supported this study.

\section{Authors' Contributions}

FO 1) designed the study, 2) coordinated and supervised the Survey on Dietary Environment in Relation to Difficulty in Shopping and Current Health Conditions of Household Elderly, 3) analyzed the data, 4) created the original research question, and 5) was a major contributor in writing the manuscript. ST completed the statistical analysis. All authors discussed, revised the manuscript, and approved the final version of the manuscript. 


\section{Acknowledgements}

Not applicable.

\section{Competing Interests Statement}

The authors declare that they have no competing interests.

\section{References}

Japanese Orthopaedic Association. (2015). Locomotive syndrome. [In Japanese]. Retrieved October 30, 2019, from https://www.joa.or.jp/public/locomo/locomo_pamphlet_2015.pdf.

Japan Osteoporosis Society, the Japanese Society for Bone and Mineral Research, \& Japan Osteoporosis Foundation. (2015). Japanese Guidelines for Prevention and Treatment of Osteoporosis 2015. [In Japanese]. Tokyo, Japan: Lifescience Publishing.

Kawaguchi, H. (2014). Molecular pathophysiology of osteoarthritis. [In Japanese]. Jpn J Clin Med, 72, 1729-1733.

Kuzuya, M. (2015). Sarcopenia: diagnosis, etiology, and intervention. [In Japanese]. Nihon Ronen Igakkai, 52, 343-349.

Ministry of Health, Labour and Welfare. (2012). Reference Materials for Promotion of Second Active Healthy Lifestyle Project table Japan 2012. [In Japanese]. Retrieved October 30, 2019, from https://www.mhlw.go.jp/bunya/kenkou/d1/kenkounippon21_02.pdf.

Ministry of Health, Labour and Welfare. (2009). Physical Activity Reference for Health Promotion: Reference for Those Aged 65 and Older 2013. [in Japanese]. Retrieved October 30, 2019, from https://www.mhlw.go.jp/stf/houdou/2r9852000002xple-att/2r9852000002xpqt.pdf.

Ministry of Health, Labour and Welfare. (2015a). Prefectural Life Tables 2015. [in Japanese]. Retrieved October 30, 2019, from https://www.mhlw.go.jp/toukei/saikin/hw/life/tdfk15/index.html.

Ministry of Health, Labour and Welfare. (2015b). National Livelihood Survey: Percent Distribution of Main Causes of Requiring Care by Care-Level Group 2015. [In Japanese]. Retrieved October 30, 2019, from https://www.mhlw.go.jp/toukei/list/d1/20-21-h28.pdf.

Ministry of Health, Labour and Welfare. (2016). Abridged Life Table 2016. [In Japanese]. Retrieved October 30, 2019, from https://www.mhlw.go.jp/toukei/saikin/hw/life/life16/index.html

Ministry of Health, Labour and Welfare. (2019). Nursing-Care Insurance Database 2016. [in Japanese]. Retrieved October 30, 2019, from https://www.mhlw.go.jp/file/05-Shingikai-12601000-Seisakutoukatsukan-Sanjikanshitsu_Shakaihoshoutant ou/0000142865.pdf.

Nakamura, K. (2008). Locomotive syndrome, disability-free life expectancy, and locomotive organ health in a "super-aged" society. Orthop Sci, 13, 1-2. https://doi.org/10.1007/s00776-008-1302-y

Nakamura, K. (2009). Locomotive syndrome in an aged-society. [in Japanese]. J Phys Med, 20, 300-304.

Nakamura, K., Yoshimura, N., Akune, T., Ogata, T., \& Tanaka, S. (2014). The concept and definition of locomotive syndrome in a super-aged society. [in Japanese]. Jpn J Clin Med, 72, 1715-1720.

Noh, J. W., Kim, H. J., Lee, C., Oh, I. H., \& Kwon, Y. D. (2016). Social activities, socioeconomic factors, and overweight status among middle-aged and older Korean adults: a cross-sectional study. Asia Pac J Public Health, 28, 157-166. https://doi.org/10.1177/1010539516628175

Okada, F., Hayakawa, F., Kugu, F., Yano, H., Toyama, M., Kitamura, H., \& Uetani, R. (2012). Survey on dietary environment in relation to difficulty in shopping and current health conditions of household elderly. [In Japanese]. Tokyo, Japan: Japanese Association of Dietary Life.

Okada, F., Toyokawa, S., Kaneko, T., \& Furuhata T. (2015a) Food purchase and locomotive syndrome among retired household elderlies -survey on dietary environment in relation to difficulty in shopping and current health conditions of household elderly-. Bull Soc Med, 33, 1-8. http://jssm.umin.jp/report/no33-2/33-2-01.pdf

Okada, F., Toyokawa, S., Kaneko, T., \& Furuhata, T. (2015b). Adverse Relationship of Locomotive Syndrome on Social Capital, Life Satisfaction, and Self-Rated Health- Survey on Dietary Environment in Relation to Difficulty in Shopping and Current Health Conditions of Household Elderly-. J Health Hum Eco, 82, 171-181. https://doi.org/10.3861/jshhe.82.171

Okada, F., Toyokawa, S., Kaneko, T., \& Furuhata, T. (2017) .Loss of access to daily necessities and locomotive 
syndrome -Nationwide Cross-Sectional Survey on Dietary Environment in Relation to Difficulty of Shopping for Elderly Living at Home. Bull Soc Med, 35, 19ᄀ28. http://jssm.umin.jp/report/no35-1/35_1_03.pdf

Rose, G. (2008). Rose's Strategy of Preventive Medicine. Oxford, NY: Oxford University Press. https://doi.org/10.1093/acprof:oso/9780192630971.001.0001

Sakurai, R., Yasunaga, M., Nishi, M., Fukaya, T., Hasebe, M., Murayama, Y., ... Fujiwara, Y. (2018). Co-existence of social isolation and homebound status increase the risk of all-cause mortality. Int Psychogeriatr, 31, 1-9. https://doi.org/10.1017/S1041610218001047

StataCorp. (2013). Stata Statistical Software: Release 13. College Station, TX: StataCorp LP.

Takahashi, H., Kawai, H., \& Kobayashi, K. (2003). Reasons for high prevalence of knee osteoarthritis in females. [In Japanese]. J Jpn Physic Therapy Assoc, 30, Supple 263.

Yoshimura, N. (2012). Clarification of factors for development of osteoporosis and osteoarthritis: general overview and follow up of long-term resident cohort. [In Japanese]. Report of Grant-in-Aid for Scientific Research (KAKENHI) 2012. Retrieved October 30, 2019, from https://kaken.nii.ac.jp/ja/file/KAKENHI-PROJECT-20390182/20390182seika.pdf

\section{Copyrights}

Copyright for this article is retained by the author(s), with first publication rights granted to the journal.

This is an open-access article distributed under the terms and conditions of the Creative Commons Attribution license (http://creativecommons.org/licenses/by/4.0/). 